Check for updates

The BMJ

Cite this as: BMJ 2021;373:n1185 http://dx.doi.org/10.1136/bmi.n1185 Published: 10 May 2021

\section{Covid-19: UK offers under 40s alternative to AstraZeneca vaccine to boost confidence}

\author{
Elisabeth Mahase
}

Adults under 40 with no underlying health conditions that put them at risk of serious covid-19 illness will be offered an alternative to the Oxford-AstraZeneca vaccine, the UK's Joint Committee on Vaccination and Immunisation has said, as long as one is available and it does not cause a substantial delay.

This decision, which is based on a risk-benefit calculation, was informed by the committee's review of the latest evidence on the AstraZeneca vaccine and the extremely rare cases of concurrent blood clots and low platelet count, as well as the current infection rates and vaccine supply plans in the UK.

Younger people with no underlying conditions are less likely to develop serious covid-19 illness than those with underlying conditions or older people, so their risk-benefit profile is different.

\section{Rare events}

Speaking at a televised conference on 7 May, the joint committee's covid-19 chair, Wei Shen Lim, said, "We offer this advice which places a high priority on safety in the hope that it will further increase vaccine confidence, so that those of us who are being offered a vaccine in the coming weeks can feel more confident and feel more able to step forward, accept the offer of a vaccine, and be vaccinated."

Lim said that this advice was specific to the current UK context and could change if vaccine uptake declined or deployment slowed. "In that scenario, any vaccine offered early is preferred to a vaccine offered too late," he said.

He added that, as the AstraZeneca vaccine required "less stringent transport and storage conditions compared to some other vaccines" in certain settings, it may be the only practical vaccine to offer. "In those circumstances, the AstraZeneca vaccine should indeed be the preferred vaccine."

All adults who have had a first dose of the AstraZeneca vaccine have also been told that they should receive their second dose as normal, as there are "no safety concerns in relation to the second dose."

The Medicines and Healthcare Products Regulatory Agency has not changed its position, emphasising that the benefits of the AstraZeneca vaccine continue to outweigh the risks of getting the vaccine in the vast majority of people.

Up to 28 April the agency had received 242 reports of blood clotting cases with low levels of platelets after having the AstraZeneca vaccine, while 28 million doses had been administered. This equates to an overall incidence of 10.5 cases in a million doses. Most of these extremely rare events occurred after the first dose. So far in the UK more than 34 million people have received a first dose of a covid-19 vaccine, meaning that the vaccine programme had prevented at least 10 ooo deaths in England as of the end of March.

A government spokesperson said, "More than 50 million vaccines overall have already been administered, and our current vaccine supply and rate of infection means we are able to take this precautionary step while remaining on track to achieve our target of offering a vaccine to all adults by the end of July."

\section{Booking system}

However, this latest advice could mean problems with the national booking system, which does not allow people to choose which vaccine they receive when booking a slot. Problems have already been reported by pregnant women, as only the Pfizer and Moderna vaccines are recommended for them in the UK. Marshall, chair of the Royal College of General Practitioners, said, "When the guidance [for pregnant women] came out, the suggestion was that the booking system would be redesigned so [that] it is possible to book for a specific vaccine if you're eligible for it.

"But that hasn't happened. We don't know why. It seems that what sounds like a pretty simple technical exercise is rather more complicated. But that really does need to happen, not only to help pregnant women now but in particular to help the larger cohorts of younger people, who will have to specify which vaccine they're going to have."

Marshall said that this left pregnant women with no choice but to ring their GP, midwife, or obstetrician to secure a slot at a certain clinic with the right vaccine. This can mean calling back many times.

"The alternative is [for GPs] to try to search for where the Pfizer or Moderna is available," said Marshall. "But that's actually a really difficult and very time consuming thing to do, and when general practice is under so much pressure, as it is at the moment, the last thing we need to be doing is ringing around trying to find the right vaccine."

Correction: On 10 May 2021 we inserted a missing "not" in the sentence "However, this latest advice could mean problems with the national booking system, which does not allow people to choose which vaccine they receive when booking a slot."

This article is made freely available for use in accordance with BMJ's website terms and conditions for the duration of the covid-19 pandemic or until otherwise determined by BMJ. You may use, download and print the article for any lawful,
Speaking to Radio 4's Today programme, Martin 
non-commercial purpose (including text and data mining) provided that all copyright notices and trade marks are retained. 